\title{
Creating identity with nature inspired lighting design - The Sensitive Organism
}

\author{
Anna-Rosa Hiort-Lorenzen¹, Beáta E. Kublik², Gordon Jäntsch ${ }^{3}$, Paulina M. Dudkiewicz ${ }^{4}$ \\ and Georgios Triantafyllidis (Supervisor) ${ }^{5}$
}

Master of Lighting Design, Department of Architecture, Design, and Media Technology, Faculty of Engineering and Science, Aalborg University Copenhagen, Denmark, 2017

\begin{abstract}
People, as living organisms, exist in symbiosis with nature; therefore, contact with nature is a basic human need. City nature is an important aspect of the urban living environment because people need an escapist experience from the everyday city life that they should find within their close environment. The outdoor lighting of urban nightscapes has a major influence on people's perception of space. Cities with an increasing number of inhabitants and late-night economic activity transform into 24hour operating cities. This means that more attention should be given to investigating the social needs of public spaces in terms of night-time urban lighting, creating experiences and identities with nature. This paper aims to emphasize the potential hidden in nature-inspired lighting as a topic for further development in the field of lighting design. Nature inspired lighting is based on a transdisciplinary approach, where terms like biomimicry, biophilia, and urban green space adopted from other subject fields than lighting influence the lighting design process in order to create a product addressing human inner connectedness to nature. The example used in this paper presents the concept of a lighting master plan called "The Sensitive Organism".
\end{abstract}

1 Anna-Rosa Hiort-Lorenzen: ahiort16@student.aau.dk

2 Beáta E. Kublik: bkubli16@student.aau.dk

3 Gordon Jäntsch: gjaent16@student.aau.dk

4 Paulina M. Dudkiewicz: pdudki16@student.aau.dk

5 Georgios Triantafyllidis: gt@create.aau.dk 


\section{Introduction}

Various studies have been carried out to examine the relationship between nature and lighting. The authors of this paper formulated conclusions based on two specific research activities, which are important for nature-inspired lighting design. The overall findings, used in the exemplary project - The Sensitive Organism, are briefly presented in this paper.

Natural scenes at exterior public spaces are perceived as having higher restorative quality. When natural objects are emphasized by light at night-time instead of man-made objects, these spaces are perceived to have more restorative quality [1], and changing the focus of the light in nightscapes can have similar effects on perceived restorativeness, fear and preference as changing the actual content of the scene. Illuminating artificial surfaces (e.g. asphalt pathways) with natural light patterns can enhance the presence of nature and, thus, increase the feeling of well-being.

Another research [2] investigated whether there is a more positive evaluation for the irregularity of a façade and daylight pattern in a social, rather than a working context. Three rendering scenes with different façade patterns were used by stereoscopic scene for virtual reality glasses in the experiment. Subjective evaluations of virtual space were recorded across six variations of façade pattern and sky types. The obtained evidence shows that pattern characteristics have an impact on the perceived spatial ambience with a preference for irregularity.

\section{State of the Art}

For nature inspired lighting, nature and its characteristics provide the main source of inspiration for the design. The aim goes beyond highlighting the existing nature in a favourable way. Such design should support the relationship between humans and nature, thus a preliminary study of basic terms describing this particular relationship is required. Three main terms, considered by the authors fundamental and relevant for the understanding of the context as well as the requirements and challenges of nature-inspired lighting, are analysed below.

a) The term Biophilia, introduced in 1973 by Erich Fromm, claims that humans possess an innate tendency to connect with nature and other forms of life [3]. Originally, Fromm described it as "the passionate love of life and of all that is alive". In 1984, Edward O. Wilson developed and popularised the idea through his work "Biophilia" and concluded that this affiliation might partly have a genetic basis [4]. Nowadays, people spend more and more time indoors, including buildings, cars and man-made parts of the environment. The lack of biophilic activities and the time spent in the nature can strengthen the disconnectedness of humans from nature. Therefore, effort must be put into the reestablishment of the relationship between humans and nature on a day-to-day basis. Previous research has proved that biophilia has a positive impact on productivity, lowering the level of stress, enhancing learning comprehension and creativity, and accelerating recovery from illness [5].

b) Biomimicry, also called biomimetics, is an approach that defines innovation as a search for sustainable solutions to human challenges in emulating nature timetested patterns and strategies [6]. This approach assumes that nature can provide 
working solutions to the sustainability problems humans caused and are now struggling with. Years of evolution and adaptation to change proved nature's strong ability to survive despite the challenges. The term "biomimicry" was coined in 1982, but was popularized by scientist and author Janine Benyus in the late $20^{\text {th }}$ century [7], who suggested looking at nature as a "Model, Measure and Mentor", that is, the largest laboratory that has ever existed and ever will. The article titled "Biomimetics: Design by Nature" published in National Geographic referred to biomimicry as the future of engineering [8].

c) Urban Green Space is a sub-part of urban open space, which contains all spaces accessible to the public within a city. A definition by Marilyn Myers from 1975 holds that as the counterpart of development, urban open space is a natural and cultural resource, synonymous with neither "unused land" nor "park and recreation areas" [9]. Urban green spaces represent a fundamental component of any urban ecosystem and include places, such as parks, woods, squares, gardens, and natural meadows as well as water elements ranging from ponds to coastal zones. Contrary to undisturbed natural areas outside the city, one of the urban nature's prominent features is its typical fragmentation into multiple small zones. Urban green areas play various roles in the urban environment. In particular, they increase the aesthetics of the city, provide space for recreation and preserve urban biodiversity. Contact with nature has a direct impact on physical, psychological, and social well-being [10] and such beneficial exposure can be provided to city inhabitants through urban green spaces.

The next step is to translate those definitions into the overall guidelines for lighting design. Biophilia tells us that people are attracted to nature. A more in-depth study of the variety of colours, shapes, materials, and movements in nature can be a source of inspiration for light projects in terms of both quality of light provided and the design of the luminaire. Innate human connection to nature places value upon nature and this can potentially add value to a design which mimics nature. Mimicking the nature with light can be an effective solution for urban areas with limited access to nature, and an interim way to offer experience and comfort to people whose biophilic needs are not fulfilled within their neighbourhood.

Biomimicry encourages the use of solutions that already exist in nature with the aim to create sustainable designs. Sustainability is a rising concern over the last decades and lighting design should address this issue, not only by achieving a watt-per-square-metre target. The LED revolution in the last decades contributes to significant energy savings, but sustainability should be considered in the entire lighting system, not only in the choice of the light source. Inspiration from biological solutions, from nano to macro scale, can possibly enhance lighting design in terms of its lifespan, maintenance, quality, aesthetics, energy consumption, and manufacturing costs.

Urban green spaces can be a canvas for lighting designers to contribute to the challenge of reconnecting people living in cities to nature. Proper lighting design should encourage people to use public green spaces, ensure the appropriate level of safety after the sunset and create a recreational atmosphere. While thinking of green areas, people tend to associate them with flora; however, it is important to consider an equally crucial part of nature, that is, fauna. Lighting design should support urban biodiversity, respecting flora and fauna as two coexisting elements of the urban ecosystem. 


\section{Project description}

\subsection{Context}

This case study is part of a master plan developed for a public housing area in Copenhagen, Denmark, called "Urbanplanen Syd". As Nordic countries are exposed to long, dark winter days, it is essential to put emphasis on the night-time lighting. The task was to create a lighting design solution that meets specific social requirements of the local community.

Urbanplanen Syd was built in the 1960s for families from a densely populated area of Copenhagen to offer more space and nature in a healthier surrounding. Apart from residential buildings, the area has a church, school, library and a park. In the past years, the initial vision of the area frayed, as it got a bad reputation in media; therefore, the Municipality of Copenhagen announced a competition to solve the problem by reshaping the area (Fig. 1).

\subsection{New Architectural Concept}

The refurbishment plan aims for a new, sustainable neighbourhood based on three core values. The first element is the Games and learning platform offering children and adolescents room for both fun and games, while also creating prerequisites for acquiring and gaining new knowledge as part of so-called "learning landscapes". The second element is the Community, a district of relations, involving both the large and close community. In the close community, the most important resource is the neighbour and there is always a possibility for people of different age, gender and ethnicity to meet on equal terms. And the third element is City nature, a green context, that is never too far to get an experience of green surroundings. Nature, in this proposal, sets the scene for the everyday life in Urbanplanen Syd.

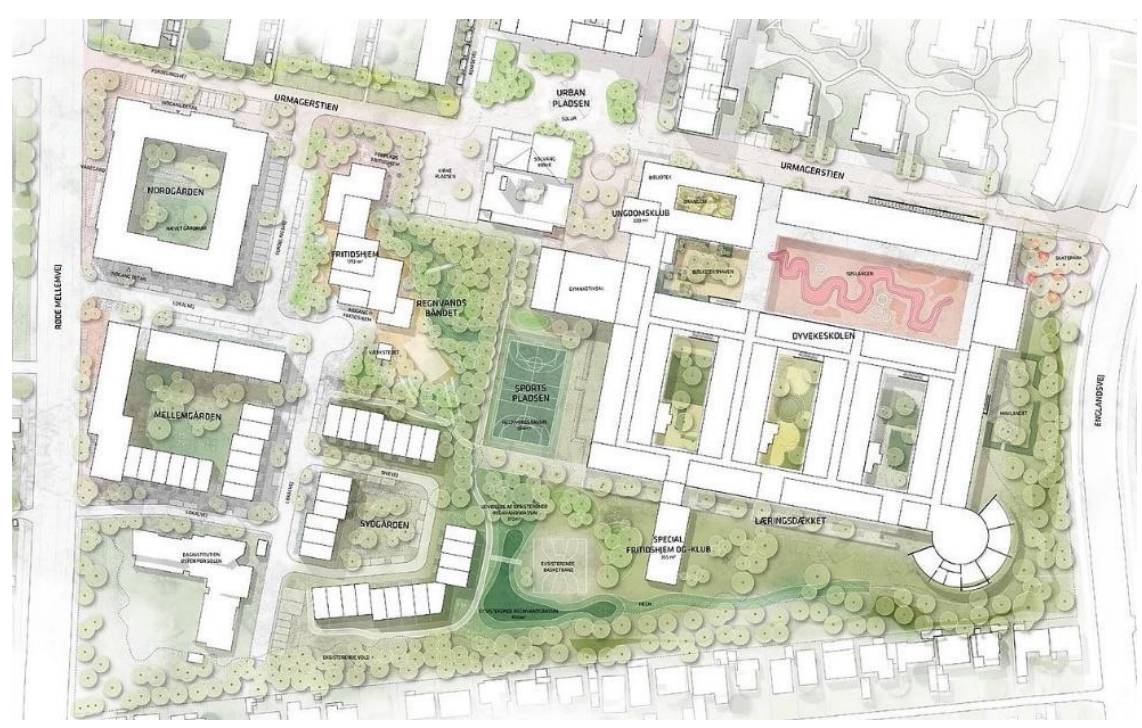

Fig 1. A new concept map introduced by the responsible architect "Tredje Natur" [11]

The task was to respond to the context and present a new architectural concept with a new, meaningful lighting design solution. 


\subsection{Problem of the Space}

The solution to the existing problem requires an in-depth investigation of the issue. The design process, in this case, involves a multi-disciplinary study of the space, its inhabitants and the architecture in the current situation, as the new plans are not applied yet.

Looking at the problems that face the space at the moment in combination with the related solutions proposed by the architects, three main issues stand out: the unused potential of nature, the deteriorating reputation and the lack of integration between different nationalities living in the area. These problems are solved with the creation of a new common identity in architecture. This perception has to be maintained in the dark hours. Lighting that brings people closer to nature can establish a recreative, escapist experience during the night for metropolitans in big cities. As this lighting project is primarily focusing on creating a meaningful lighting experience, attention should be paid to involving people in a qualitative research based on what they consider pleasant, nature-driven stimuli in a public space.

\section{Design Research}

The concept is built upon criteria that focus on the values of city nature through the creation of a nature-driven light experience by researching the appropriate qualities of nature that can be transformed into light qualities. Based on the criteria, the design research focuses on the analysis of nature-inspired lighting solutions and natural phenomena in order to get a better understanding of the following design parameters:

- Natural textures

- Organic patterns

- Natural light sources

- Natural movements

- Interaction with people

- Environmental interactions

The project refers to theories formulated by William Lam and William H. Whyte, using their guidelines in the design selection. Lam describes the functional and biological needs of people - orientation, discernibility and communication [12]. Whyte introduces the term of triangulation - a process by which some external stimulus provides a linkage between people and prompts strangers to talk to each other. This stimulus can be a physical object or sight (musician, performer, sculpture) [13].

Several urban lighting design projects aimed to improve the perception of public spaces. The Dutch lighting project called Broken Light helped change the reputation of a neighbourhood in Rotterdam by involving nature and dynamic light into the light experience [14]. By researching how light is perceived and experienced in public spaces beyond street lighting regulations, it showed that a lighting concept is not just a matter of perception, but also an enabler for the identity of a space.

The transformation was beyond aesthetic - illuminating horizontal and vertical surfaces, and creating a natural "underwater-like" moving pattern on the street, helped to tackle the issues of crime and prostitution that used to demote the neighbourhood through a strengthened sense of community.

\section{Lighting Concept}

Next, a short description of the specific master plan is provided to demonstrate how nature 
inspired lighting design might be applied to a specific area. Considering that The Sensitive Organism was only developed as a concept, all presented solutions are theoretical and have not yet been scientifically tested. In general, this master plan provides guidelines for a specific residential area in Copenhagen, but is also appropriate for other areas with similar conditions.

\subsection{The Sensitive Organism}

The concept arises from the organic shape of the area, envisioned as a structure which reacts to incentives, that is, a sensitive organism. It cares for people's needs, supports their activities and occasionally comes with surprises for the inhabitants. Even though it is an abstract structure, it has three beating hearts in the centre of the area, it has veins along the main flows of people and it has organs in every place people gather and locate their activities.

The Sensitive Organism, as a visionary guideline, helps define the key aspects of the master plan and ensures the conceptual consistency, which is important for building a strong identity through the light.

\subsection{The Raindrop Pattern}

Guided by this nature inspired vision, the main visual feature of the lighting design was adopted from the movement occurring when raindrops encounter a water surface (Fig 2). The emerging, randomly placed, shapes create a dynamic pattern of multiple concentric circles with its own peculiar rhythm.

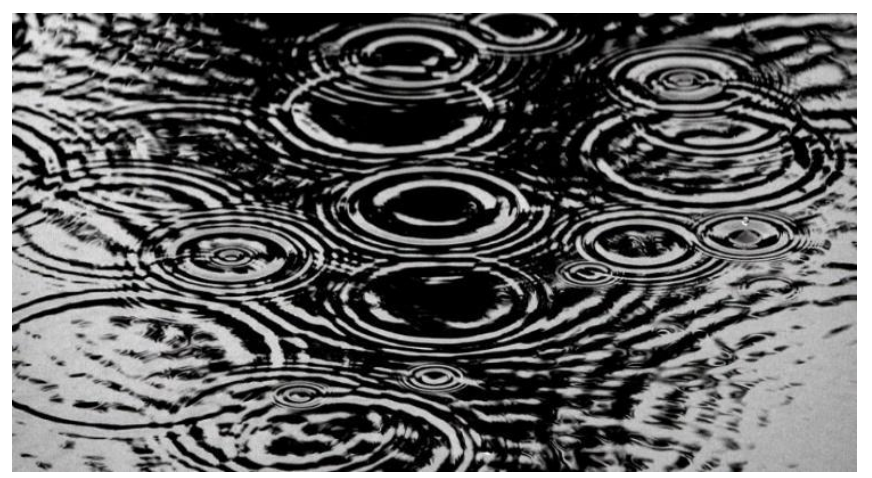

Fig 2. Typical raindrop pattern on a water surface

Translating this pattern into a light language, the shape and rhythm define the spatial light distribution. Thereby, every concentric ripple caused by a drop is represented by a spotlight from a luminaire above. According to the ripple propagation, the spot radius and brightness follow the same rhythm of the fading concentric wave. That means a static mounted spotlight projects a spot on the ground. The initial diameter is small and gradually expands within a specific time frame. While the diameter is increasing, the brightness is decreasing until zero. Finally, the small initial diameter quickly fades in and the cycle starts again. Unlike the rain, where one falling drop creates a series of concentric circles of different diameters, visible at once, one luminaire can produce only one circle at the time. Unlike the real rain pattern, only a few spots increase their radius, while their brightness is fading out to avoid an inappropriate, exaggerated visual dynamic. Most of the spots are at rest. To ensure a proper balance and create a pleasant natural, yet dynamic pattern, the spots have different initial rest diameters (Fig. 3). There is only a determined amount of used diameters extracted from the Fibonacci 
series of numbers [15]. This specific range is often found in natural growth processes or proportions. The chosen diameters are 3, 5, 8 and 13 meters which are used for the patterns.

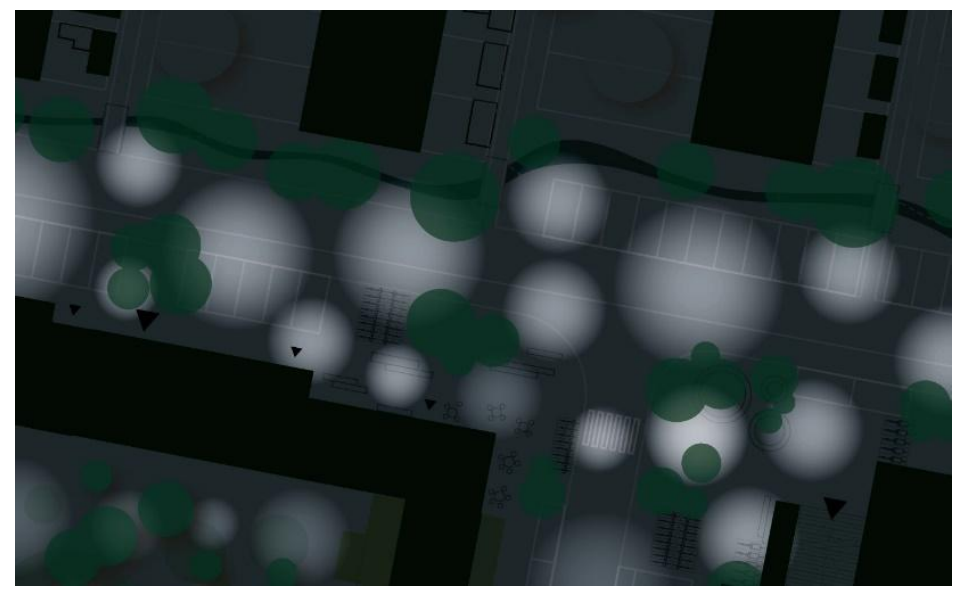

Fig 3. An example of the initial raindrop spot pattern

\subsection{Differentiated Parts of the Area}

The raindrop pattern is used for the whole area but in different variations depending on the sub-area. These sub-areas are defined by specific features corresponding to the needs of the city dwellers. These features or how they are structured and used by people create the unique atmosphere of the area. At night, the light can enhance these effects and, thus, underline its identity. In this example, there are five sub-areas, namely major and minor paths, as well as major and minor squares and parks.

The atmosphere of the paths areas is characterized by its liveliness, a high degree of communication and, therefore, exudes a higher brightness.

The squares combine a public and private atmosphere. In addition, the main squares also have attraction points, like a fountain or an art installation, to entice people. At the same time, these places have more private parts at the periphery, where people could observe each other or engage in a conversation. The minor areas for both paths and squares are always a bit more private than the major ones.

In turn, the parks are defined in this example as having the most private and relaxing atmosphere due to the fact that they have the biggest amount of natural elements.

\subsection{Parameters of a Pattern}

Each of the sub-areas is assigned an appropriate pattern in order to enhance their atmosphere. Thereby, the patterns are distinguished by the composition of the following features:

A: A range of initial spot diameters used in one pattern

B: Density of spots in the entire pattern

C: The initial brightness level of entire pattern

D: The luminous texture of the spot area in the entire pattern

Regarding A, three different ranges of diameters are used to create three kinds of patterns. The first pattern consists of all diameters $(13,8,5,3$ meters), the second excludes the smallest 
diameter (13, 8, 5 meters) and the third exclude the biggest diameter (8, 5, 3 meters). In this way, it is possible to achieve different scales of patterns. The bigger scale is suitable for a more public atmosphere whereas a smaller one is better for a cosy atmosphere [16]. Furthermore, as mentioned above the spots in some areas are dynamic. However, the biggest diameter of each range always remains static in order to ensure a good spatial orientation.

Regarding B, the density of spots corresponds to the distance between the spots within the entire pattern. Two different levels of density are used: a high level, corresponding to the distance of five meters, and a low level of three meters. As a result, a high-density pattern has a higher uniformity of illumination than a lower-densed one. Higher uniformity is suitable for a more public atmosphere, whereas lower uniformity better suits a cosy atmosphere [16].

Regarding C, two levels of horizontal illuminance in the entire master plan are used for a pattern that separates predominantly public areas from primarily private areas. A high illuminance level is assigned to public spaces and a low level to private ones. The high level should not exceed 10 lux. The existing scientific research points to the fact that the feeling of security increases with an average increase in the lighting level up to ca. 30 lux and that a small increase in illuminance up to 10 lux results in a large increase in perceived security [17].

Regarding D, the luminous texture of a spot describes the distribution of lighter and darker parts within the spot surface. Three different spot surfaces are used: a plain one, which contains no texture; a rippled texture similar to the ripples of a raindrop wave; and an organic texture with a caustic appearance (Fig. 4). The plain and rippled textures are used for the whole pattern, whereby the latter one creates a cosier atmosphere. The organic texture is only used for a single spot to serve as an eye-catcher.

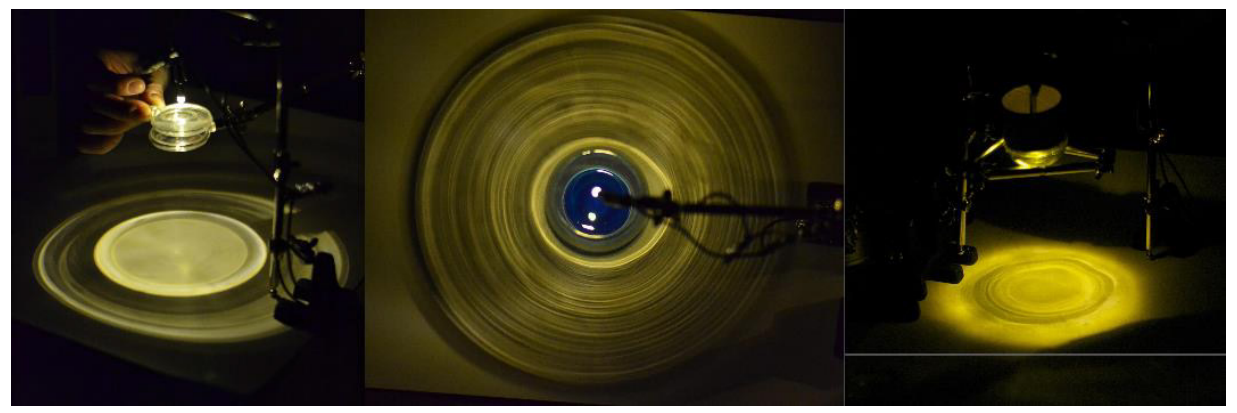

Fig 4. Three textures used: plain, ripple and organic

\subsection{Further Variations of a Pattern}

Other conceivable possibilities to adapt the pattern in order to enhance the appropriate atmosphere are the colour temperature and the height of the luminaire. A slow fade from a high to a low colour temperature may signalize the change from twilight until night and even at night, it can support a better temporal orientation.

The height of the luminaires can be adapted to the initial diameters of the spots. Whereby, the smaller the spot the lower the height is. The height of a light source towards the eye level influences the feeling of public sphere and privacy [18].

Furthermore, the speed of fading during one cycle of the raindrop rhythm can separate a quiet area from a lively one. 


\subsection{Application of the Patterns to the Areas}

Major and minor paths are the veins of the Sensitive Organism. The big light spots of the pattern are situated in the middle of the street and the smaller ones in the periphery to illuminate elements like benches, bike parking, trees and facades. Trees and facades are illuminated to enhance the verticals.

Major and minor squares are the organs, whereby the attractions situated on the major squares are the hearts of the Sensitive Organism. Although the major squares have a very public atmosphere and, thus, require a high brightness, the chosen level of brightness is low in order to enhance the contrast with the bright attraction elements. These special elements are lit by one organic spot in order to provide a contrast with the texture.

The parks are intended to be the lungs of the Sensitive Organism. With a lower brightness of the pattern, lower density and the choice for smaller diameters and rippled texture, these areas are expected to have the greatest contrast with the previous ones. They create the most private and intimate place for people.

In Table 1, an overview of all pattern parameters is shown in order to illustrate the change from public to private atmosphere from left to right.

Table 1. Overview pattern parameters according to the areas

\begin{tabular}{|l|c|c|c|c|c|c|}
\hline & \multicolumn{7}{|c|}{ Sub areas } \\
\hline Feature & $\begin{array}{l}\text { Major } \\
\text { paths }\end{array}$ & Minor paths & $\begin{array}{c}\text { Major } \\
\text { squares }\end{array}$ & $\begin{array}{c}\text { Minor } \\
\text { squares }\end{array}$ & $\begin{array}{c}\text { Attractions } \\
\text { on squares }\end{array}$ & Parks \\
\hline A: Range & $13-8-5$ & $13-8-5-3$ & $13-8-5$ & $13-8-5-3$ & 13 & $8-5-3$ \\
\hline B: Density & high & low & high & low & - & low \\
\hline C: Brightness & high & low & low & low & high & low \\
\hline D: Texture & plain & plain & plain & plain & organic & ripple \\
\hline
\end{tabular}

\subsection{Horizontal and Vertical Illumination}

Another recommendation of the master plan is the illumination of the whole field of view in order to enhance the readability of the space. This includes the illumination of horizontal as well as vertical surfaces of the environment.

The horizontal plane, involving the streets and pavements, is characterized by the raindrop pattern. It evenly covers the whole plane and is not coordinated with the geometry of the street grid. The aim is to create a natural organic view without emphasizing the straight built environment.

The vertical planes are formed by facades, trees and bushes. They are illuminated by grazing light. At the same time, natural elements are enhanced, which also increases the feeling of security [1].

\subsection{Fixtures and Mounting}

For this concept customized fixtures are necessary. Almost each fixture needs to be able to change its beam angle dynamically. This technology is already used in stage luminaries. Due to the relatively narrow beam angles, it might be possible to make the luminaires glare free. This technique is important to guide the view of the pedestrian on the floor rather than in the 
direction of the luminaire. This would be a vast change in the perception of public lighting, as nowadays street lighting is mostly not able to achieve this.

Furthermore, to generate different light textures, the fixtures should be equipped with specially treated lenses or gobos.

Due to multiple fixtures, an installation on tensed horizontal wires across the area reduces the number of polls. In addition, it provides a higher flexibility in the alignment of the fixtures in order to achieve the desired patterns.

In order to keep the maintenance of the luminaires low, it is recommended to use longlife LEDs and to avoid possible mechanical movement of lenses. Here further development steps are necessary.

\subsection{Interaction Scheme}

The Sensitive Organism was developed to create a natural identity at night in order to encourage passers-by to stay longer outside and enjoy contact with each other. People talking on the street may attract others to join the conversation. Supporting this behaviour also for people approaching from a bit more far away light is able to signalize speaking people to them. Using the dynamic light cycle of the raindrop idea in between a static light pattern may attract people because moving light always attracts attention.

By equipping the luminaire with a sound sensor, it will be possible to track talking people and trigger the dynamic drop cycle. After tracking, with the help of an interlinked controlling system, the adjacent spots start to run the cycle, too, in between a radius of about 20 meters. Once these talking people leave the place, the dynamic light fades away into the initial static pattern.

But the organism does not only react to people's behaviour but also to seasonal changes of the natural light in order to create a harmonic transition from day to night. The twilight duration can influence the fading time of the whole artificial light after sunset. Even the colours of the sunset can control the colours of the patterns during the night. To elaborate on this topic, further investigations are necessary and can be discussed in another paper.

\subsection{Lighting Concept Summary}

In conclusion, to illuminate a space in order to create natural identity the concept makes use of the idea of patterns, following the Fibonacci row, textures similar to ripples and organic caustics and natural rhythms, expressed by cycles of dynamic spot diameters and brightness.

To generate a real-time sensitivity to the organism concept, an interaction scheme can be implemented to influence the behaviour of the pedestrians.

\section{Conclusion}

The project "The Sensitive Organism" was an attempt to use light as a tool for enhancing the city nature and thus bringing inhabitants closer to each other by giving them space with a strong identity during night-time. This paper reflects substantial interest in the topic, where a case project was presented mainly to inspire the reader for its own further investigation, as the main focus should be placed on the overall potential of highlighting the nature in our design. Besides being aesthetic, nature-inspired lighting design can address human biophilic needs of having contact with nature on a daily basis. It can be done directly, by leading people with light into green spaces within the urban environment, or indirectly by mimicking nature with light, like "The Sensitive Organism" project did with the rain ripples. Cities are getting bigger and nature is often polluted, so light is an "easy" way to create natural experience and 
change appearance without any significant intervention into architecture. Biomimicry goes even further by looking at nature as a source of solutions that are not only pleasant for people but are sustainable, of high quality and can last long, as nature proved to be able to survive all kind of changes including environmental challenges.

Benefits offered by nature are unlikely to be realized without a conscious decision of designers to enhance the presence of nature in cities and connect people to the surrounding nature.

\section{Acknowledgement}

The lighting design "The Sensitive Organism" was created as a group semester project in the framework of the lighting design master programme at Aalborg University Copenhagen in collaboration with the municipality of Copenhagen, tredje architecture and jaja architecture Copenhagen, who provided the authors with the material for the residential area of Urbanplanen Syd in Copenhagen. 


\section{References}

[1] Heli Nikunen \& Kalevi M. Korpela, The effects of scene contents and focus of light on perceived restorativeness, fear and preference in nightscapes, Journal of Environmental Planning and Management, Vol 55, No.4, 453-468, 2012

[2] Kynthia Chamilothori, Jan Wienold \& Marilyn Andersen. Daylight patterns as a means to influence the spatial ambiance: a preliminary study, Interdisciplinary Laboratory of Performance-integrated Design (LIPID) Ecole polytechnique fédérale de Lausanne (EPFL), Switzerland, 2016

[3] biophilia, https://www.britannica.com/science/biophilia-hypothesis, downloaded December 2017

[4] E.O. Wilson, Biophilia, Harvard University Press, 1984

[5] Kellert, S. R., Heerwagen, J., \& Mador, M. L. Biophilic Design: The Theory, Science, and Practice of Bringing Buildings to Life, published 2008

[6] Biomimicry, https://biomimicry.org/, downloaded December 2017

[7] What is Biomimicry, http://environment-ecology.com/biomimicry-bioneers/367what-is-biomimicry.html, downloaded December 2017

[8] Biomimetics, http://ngm.nationalgeographic.com/2008/04/biomimetics/tom-muellertext, downloaded December 2017

[9] Marilyn. "Decision Making in Allocating Metropolitan Open Space: State of the Art." Transactions of the Kansas Academy of Science 1975. pp 149-153.

[10] Keniger et al. 2013, Hough 2014

[11] Tredje Natur; https://www.urbanplanen.com/urbanplanen-syd?lightbox=dataItemj03v25r6, downloaded December 2017

[12] Lam, William M., C., "Perception and Lighting as form-givers for Architecture",1977

[13] Whyte, William.H, "The Social Life of Small Urban Spaces" 1980

[14] "Broken Light”, https://www.alanod.com/en/projects/broken-light/, downloaded 2017

[15] Fibonacci, http://www.maths.surrey.ac.uk/hosted-sites/R.Knott/Fibonacci/fibnat. html , downloaded 2017

[16] Lam, William M., C., "Perception and Lighting as form-givers for Architecture",1977

[17] Boyce PR, Eklund NH, Hamilton BJ, Bruno LD. Perceptions of safety at night in different lighting conditions. Lighting Research and Technology 2000; 32: 79-91.

[18] Descottes Hervé, "Architectural Lighting. Designing with Light and Space". Princeton Architectural Press, NY, 2011 